\title{
The association between metabolic syndrome and lower urinary tract symptoms suggestive of benign prostatic hyperplasia in aging males: evidence based on propensity score matching
}

\author{
Yang Xiong ${ }^{1,2}$, Yangchang Zhang ${ }^{3}$, Jun Tan ${ }^{1,2}$, Feng Qin ${ }^{1}$, Jiuhong Yuan ${ }^{1,2}$ \\ ${ }^{1}$ Andrology Laboratory, West China Hospital, Sichuan University, Chengdu, China; ${ }^{2}$ Department of Urology, West China Hospital, Sichuan \\ University, Chengdu, China; ${ }^{3}$ Department of Epidemiology and Health Statistics, School of Public Health and Management, Chongqing Medical \\ University, Chongqing, China \\ Contributions: (I) Conception and design: Y Xiong; (II) Administrative support: F Qin, J Yuan; (III) Provision of study materials or patients: Y Xiong; \\ (IV) Collection and assembly of data: Y Xiong, Y Zhang; (V) Data analysis and interpretation: Y Xiong, Y Zhang, J Tan; (VI) Manuscript writing: All \\ authors; (VII) Final approval of manuscript: All authors. \\ Correspondence to: Prof. Feng Qin. Andrology Laboratory, West China Hospital, Sichuan University, Chengdu 610041, China. \\ Email: 174299227@qq.com; Prof. Jiuhong Yuan, Andrology Laboratory/Department of Urology, West China Hospital, Sichuan University, Chengdu \\ 610041, China. Email: jiuhongyuan2107@163.com.
}

Background: To investigate the association between lower urinary tract symptoms suggestive of benign prostate hyperplasia (LUTS/BPH) and metabolic syndrome (MetS) in aging Chinese males.

Methods: A dataset that included 3,568 non-MetS cases and 1,020 MetS cases (after data cleansing) was downloaded from the China Health and Retirement Longitudinal Study (CHARLS). To balance the intergroup covariates, propensity score matching (PSM) was employed in the analyses. Univariate logistic regression and multivariate logistic regression were then performed to investigate the relationship between LUTS/BPH and MetS in aging Chinese males.

Results: Before PSM, multivariate logistic regression showed that participants with MetS had a 1.47 times higher risk of LUTS/BPH compared to non-MetS cases in the final model $(\mathrm{P}<0.001)$. It also revealed that participants with low high-density lipoprotein (HDL), abdominal adiposity, or high triglycerides had a higher probability of LUTS/BPH [odds ratio (OR) =1.56 for low HDL; OR =1.50 for abdominal adiposity; and $\mathrm{OR}=1.48$ for high triglyceride, $\mathrm{P}<0.001$ ], while participants with hyperglycemia or hypertension had identical odds of LUTS/BPH ( $>0.05)$. After PSM, 1,000 pairs were successfully matched. It was also found that MetS cases had a 1.60 times higher risk of LUTS/BPH compared to non-MetS cases $(\mathrm{P}<0.001)$, and participants with low HDL, abdominal adiposity, high triglycerides, or hyperglycemia had a higher likelihood of LUTS/BPH than their counterparts $(\mathrm{P}<0.001)$. However, the probability of LUTS/BPH in hypertensive patients remained similar to that in non-hypertensive patients $(\mathrm{P}>0.05)$.

Conclusions: Aging Chinese males with MetS had a higher probability of LUTS/BPH. Also, patients with low HDL, abdominal obesity, high triglycerides, or hyperglycemia had an increased risk of LUTS/BPH; however, this was not the case for hypertensive patients.

Keywords: Metabolic syndrome (MetS); benign prostatic hyperplasia (BPH); lower urinary tract symptoms (LUTS); aging males; propensity score matching (PSM)

Submitted Jul 28, 2020. Accepted for publication Nov 06, 2020.

doi: $10.21037 /$ tau-20-1127

View this article at: http://dx.doi.org/10.21037/tau-20-1127 


\section{Introduction}

Benign prostatic hyperplasia (BPH), which is characterized by the nonmalignant and unregulated growth of the prostate gland, remains highly prevalent in elderly men. An enlarged prostate contributes to bladder outlet obstruction and induces symptoms such as frequent voiding, urgency, nocturia, and urine retention, among others (1). It is the leading cause of lower urinary tract symptoms (LUTS) which can include weak urinary stream, increased urgency, frequent urination, nocturia etc., and is a heavy burden on the elderly population worldwide. Thus, there is a pressing need for effective treatment of this condition (2).

According to one recent study, the prevalence of LUTS/ $\mathrm{BPH}$ in aging Chinese males is $11.97 \%$, and is $22.70 \%$ in participants aged 70 years old and above (3). Yue et al. reported that the prevalence of $\mathrm{BPH}$ ranges from $2.17 \%$ in men aged $40-44$ years to $31.11 \%$ in men aged 80 years and above (4). In America, this disease affects an estimated 15 million men aged 30 years and older (5). Considering the high prevalence and significantly adverse effects for males, identifying factors associated with LUTS/BPH is critical, and is the aim of the present study. Throughout the longterm exploration of the factors associated with LUTS/BPH, numerous risk factors have been identified, including age, hyperlipidemia (6), and hypogonadism (7), among others. Various studies have scrutinized metabolic syndrome (MetS) to investigate its association with LUTS/BPH. Preliminary research has found that the close relationship between these conditions may be mediated by systemic inflammation and oxidative stress (8), which are induced by MetS and subsequently enhance the incidence of LUTS/BPH $(9,10)$.

MetS is defined by five components including visceral adiposity, high blood glucose (fasting), disordered lipid metabolism [elevated blood triglycerides and decreased high-density lipoprotein (HDL)], and hypertension. Over the past two decades, the prevalence of MetS has increased worldwide, especially in the elderly population (11). A previous study has reported that the overall prevalence of MetS is $33.9 \%$ and affects approximately 454 million Chinese people (12). Both diseases (LUTS/BPH and MetS) are commonly seen in the aging population. Several studies have reported inconsistent conclusions regarding the relationship between MetS and LUTS/BPH. One prospective study by Yue et al. revealed that MetS may accelerate the clinical progression of $\mathrm{BPH}$ in middle-aged and older men (13). The conclusions reached by some other studies were also in line with Yue's study (14-18). However, a study that examined Korean men aged 50 years old proposed a conflicting conclusion (19), and relevant studies performed in Chinese (20), Japanese (21), and American (22) populations echoed this finding. These inconsistent results reveal a positive association between LUTS/BPH and MetS, and thus, more evidence is required that accounts for different countries, ethnicities, economic status, and other factors.

In the present study, a representative dataset targeting the elderly population was downloaded from the China Health and Retirement Longitudinal Study (CHARLS) to explore the association between LUTS/BPH and MetS. To balance intergroup discrepancies in the covariates, propensity score matching (PSM) was adopted to perform further analyses. We present the following article in accordance with the STROBE (Strengthening The Reporting of Observational Studies in Epidemiology) reporting checklist (available at http://dx.doi.org/10.21037/tau-20-1127).

\section{Methods}

\section{Data collection and study samples}

The dataset targeting aging Chinese males (aged 40 years and above) was downloaded from the CHARLS official website (http://charls.pku.edu.cn/), which is a representative national survey initiated in 2008. This project collected data gathered by well-trained researchers from 28 provinces and 150 counties in China. On April 23, 2020, we downloaded the CHARLS Follow-up Questionnaire (2015) to evaluate the association between LUTS/BPH and MetS.

Data collection was performed by medically trained staff from the Chinese Center for Disease Control and Prevention (China CDC). One $4 \mathrm{ml}$ tube of venous blood was collected from each respondent for testing blood biomarkers like blood glucose, HDL, low-density lipoprotein (LDL), and blood triglycerides. Participants were asked to fast overnight; however, researchers took blood even if they were not fasting and then noted their status. In further analyses, participants who had not fasted were excluded according to the International Diabetes Federation (IDF) definition of MetS. After the venous blood was separated into plasma, the plasma was stored in three $0.5 \mathrm{~mL}$ cryovials. These cryovials were then immediately stored at $-20^{\circ} \mathrm{C}$ and transported to the Chinese CDC in Beijing within 2 weeks. Here, they were placed in a deep freezer and stored at $-80{ }^{\circ} \mathrm{C}$ until they were assayed at the 


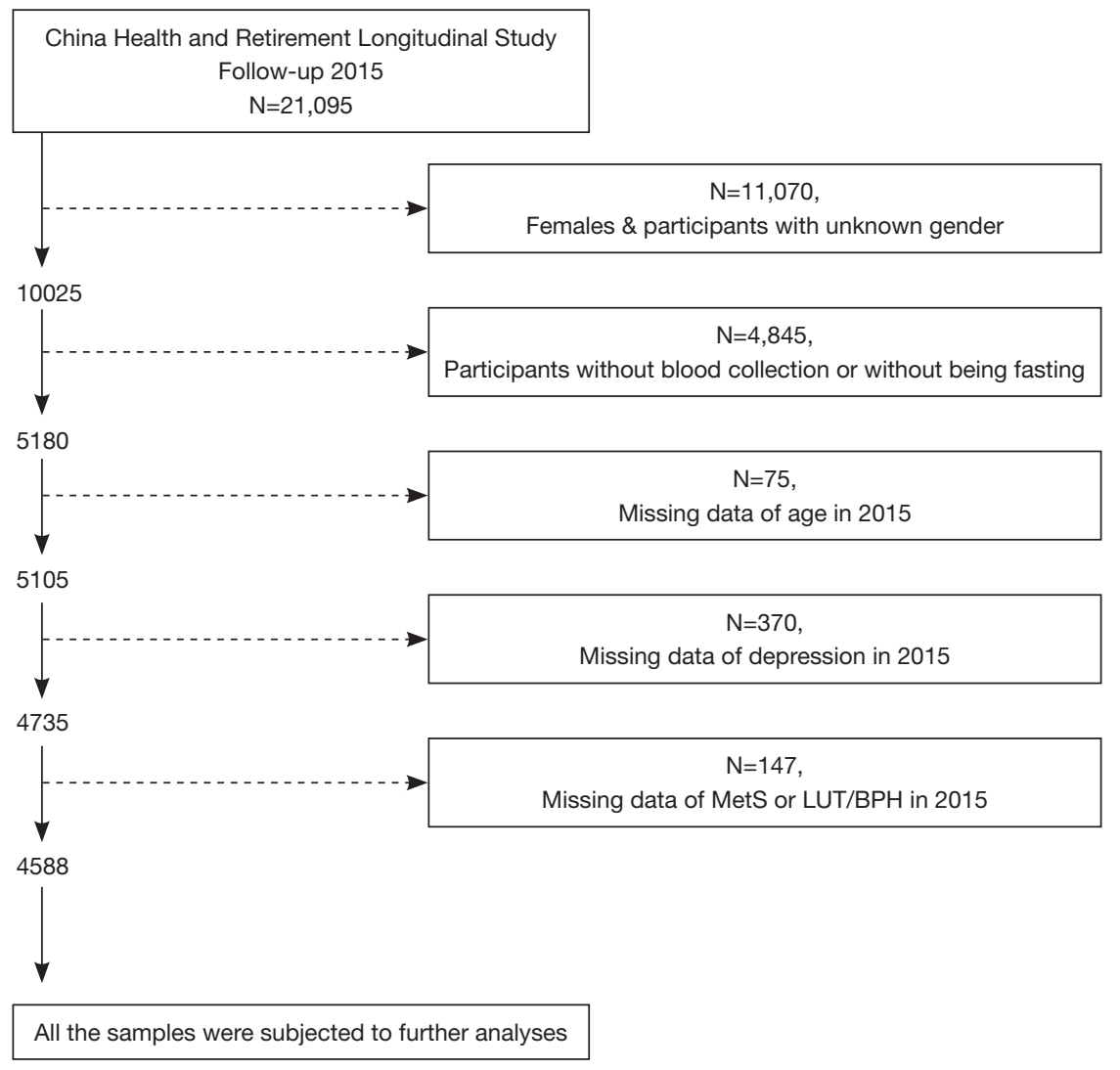

Figure 1 Data cleansing flow-chart.

Capital Medical University laboratory using an enzymatic colorimetric test. Researchers also collected relevant medication history for treating diabetes, dyslipidemia, and hypertension, but did not record any specific drug names.

To measure waist circumference, participants were asked to stand still, take a normal breath, and then exhale and hold their breath at the end of their exhalation. The researchers placed measuring tape over the clothing around the waist at the level of the navel and recorded the measurement. For blood pressure, participants were asked to be relaxed, remain seated and quiet during the measurements, and then to lay their arms on a flat surface with the palm facing up so that the center of their upper arms were at the same height as their hearts. Each person's blood pressure was measured three times, and the average of the three measurements was taken as the final blood pressure. A more detailed description of CHARLS has been provided in a previous publication (23).

All procedures performed in this study were in accordance with the Declaration of Helsinki (as revised in 2013) and were approved by the Ethics Committee of Peking University (IRB 00001052-11014). Written and oral informed consent was obtained from all participants prior to their enrollment in this study. In the present study, after clearing the missing values of LUTS/BPH and MetS in aging males, a total of 4,588 participants were enrolled. The flow chart of data cleansing is shown in Figure 1. To balance the discrepancies between covariates in the two groups, PSM was employed with 1,000 pairs matched successfully.

\section{Covariates}

Covariates including age, marital status, depression status, sleeping time, afternoon napping, geographical region, smoking, alcohol consumption, LDL, total cholesterol (TC), and uric acid were collected. Age was divided into four groups: $40-50,50-60,60-70$, and $>70$ years. Marital status was organized into two parts. The first part involved married couples living together and cohabiting individuals; the second part included people who are married but not 
living with their spouse, in addition to separated, divorced, widowed, and never-married individuals. Sleeping time consisted three groups: $0-6$ hours, $6-8$ hours, and $>8$ hours. Geographic region included rural and urban. Smoking was divided into yes, no, and previous smoker. Alcohol consumption was divided into three categories: more than once a month, less than once a month, and never. In CHARLS, the Epidemiological Studies Depression Scale-10 (CESD-10), a widely used questionnaire, was adopted to assess depressive state (24). Participants with total scores $\geq 10$ were defined as depressed. Afternoon napping was classified as yes and no. LDL was divided into two groups: $\leq 120$ and $>120 \mathrm{mg} / \mathrm{dL}$ (25). TC was categorized as $\leq 200$ and $>200 \mathrm{mg} / \mathrm{dL}$ (26). Uric acid was divided into two groups: $\leq 420 \mu \mathrm{mol} / \mathrm{L}$ (non-hyperuricemia) and $>420 \mu \mathrm{mol} / \mathrm{L}$ (hyperuricemia) (27).

\section{Definition of MetS and LUTS/BPH}

Three principal definitions of MetS offered by the World Health Organization (WHO), the National Cholesterol Education Program (NCEP), and the IDF have been adopted globally. In our study, the IDF criteria were used due to their suitability for the Chinese population and low cutoff values. This sensitive set of criteria facilitate early identification and intervention. Participants were defined as MetS when they exhibited visceral adiposity (waistline $>90 \mathrm{~cm}$ ) along with the presence of two or more of the following criteria: (I) fasting blood glucose greater than $100 \mathrm{mg} / \mathrm{dL}(5.6 \mathrm{mmol} / \mathrm{L})$ or diagnosed diabetes; (II) HDL cholesterol $<40 \mathrm{mg} / \mathrm{dL}(1.0 \mathrm{mmol} / \mathrm{L})$ in men or those with drug treatment for low HDL cholesterol; (III) blood triglycerides $>150 \mathrm{mg} / \mathrm{dL}(1.7 \mathrm{mmol} / \mathrm{L})$ or drug treatment for elevated triglycerides; and (IV) blood pressure $>130 / 85 \mathrm{mmHg}(17.33 / 11.33 \mathrm{kPa})$ or drug treatment for hypertension. As for LUTS/BPH, participants were evaluated with the following question: "Have you ever been diagnosed with a prostate illness, such as prostate hyperplasia, excluding prostate cancer?" Relative symptoms were interpreted for the participants, and included dysuria, nocturia, intermittent urinary stream, and slow urinary stream, among others. BPH did not occur with LUTS simultaneously for one hundred percent. Only when these patients perceived the symptoms stated above, would they seek medical assistance, and thus, subjects that answered with a definite "Yes" during the survey were defined as having LUTS/BPH.

\section{Statistical analysis}

Data are presented according to their types. Categorical data are presented as proportions (\%), while continuous data are presented as mean \pm standard deviation (SD). Discrepancies between baseline characteristics were assessed using the chi square test or $t$ test according to the types of data. Before PSM, univariate and multivariate logistic regression were adopted to explore the association. Furthermore, PSM (nearest-neighbor matching with caliper) was used, and discrepancies between covariates were balanced at ratio of 1:1 with no replacement matching and a caliper value of 0.03 . After PSM, univariate logistic regression was used to explore the difference between the MetS and non-MetS groups. All of the data analyses were performed using STATA 15.0 (Stata Corporation, College Station, TX, USA). All the figures were constructed using GraphPad Prism 8.0 version (GraphPad Software Inc., San Diego, CA, USA). A P value $<0.05$ (two-sided) was considered statistically significant.

\section{Results}

\section{Baseline characteristics of participants}

A total of 4,588 participants were enrolled. The baseline characteristics of the participants are displayed in Table 1 . Subjects were divided into a MetS group and non-MetS group with 3,568 and 1,020 participants, respectively. Before PSM, the covariates were lopsided $(\mathrm{P}<0.05)$, except for alcohol consumption and LDL $(\mathrm{P}>0.05)$. After PSM, 1,000 pairs were successfully matched with 11 balanced covariates.

\section{PSM result confirmation}

To confirm that the covariates of PSM were well balanced, a histogram of the propensity scores in the treated group (MetS group) and the untreated group (non-MetS group) was constructed (Figure $2 A, B$ ). It was clear that the distribution of propensity scores became symmetrical when Figure $2 A$ was compared with Figure 2B. Furthermore, the absolute standardized differences in Figure $2 C$ indicated that all the differences were less than $5 \%$ (differences $<10 \%$ were considered as adequate matching). These results demonstrated that the discrepancies between covariates were amply balanced by PSM, which allowed us to perform further analyses. 
Table 1 Baseline characteristics for participants

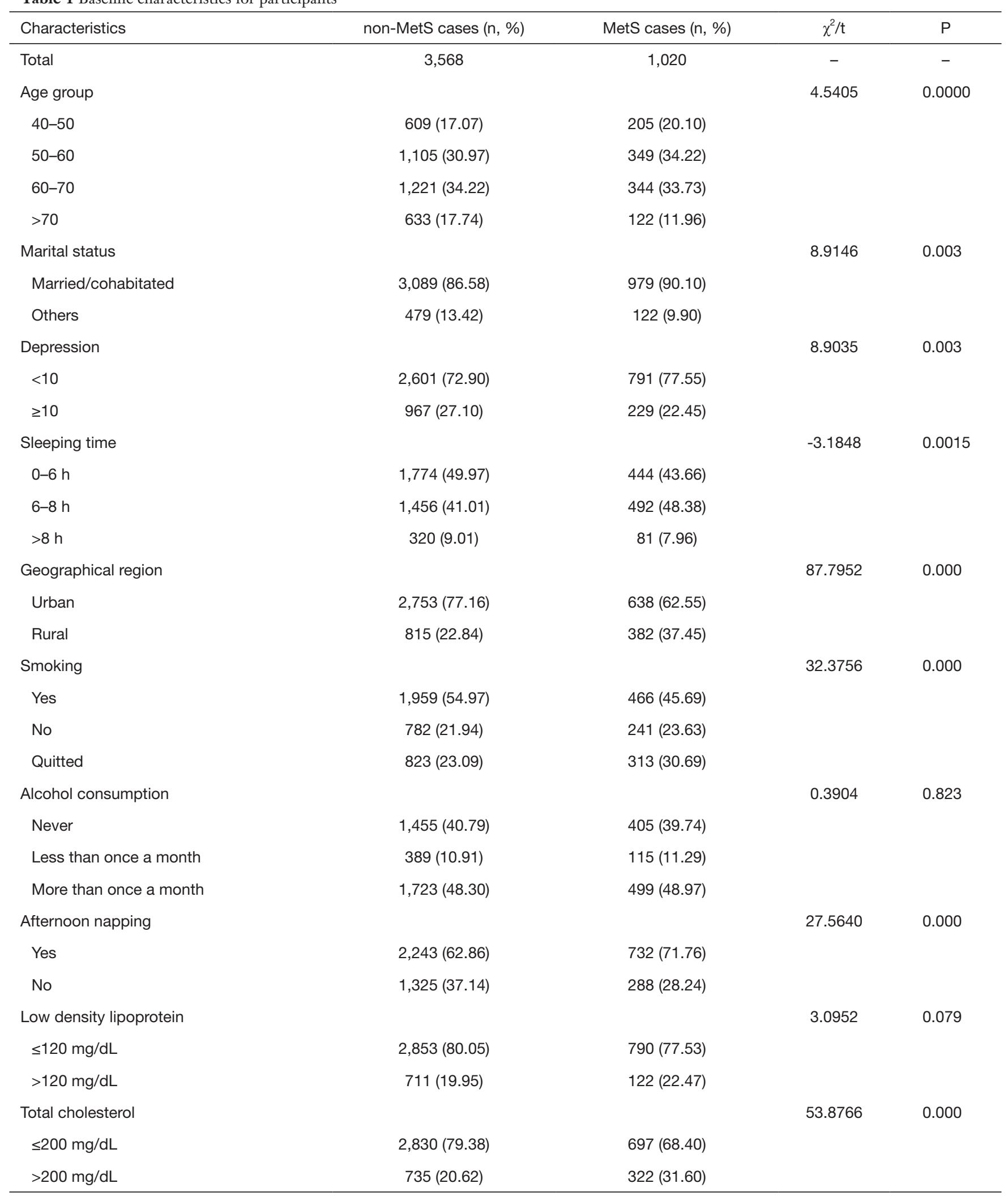

Table 1 (continued) 
Table 1 (continued)

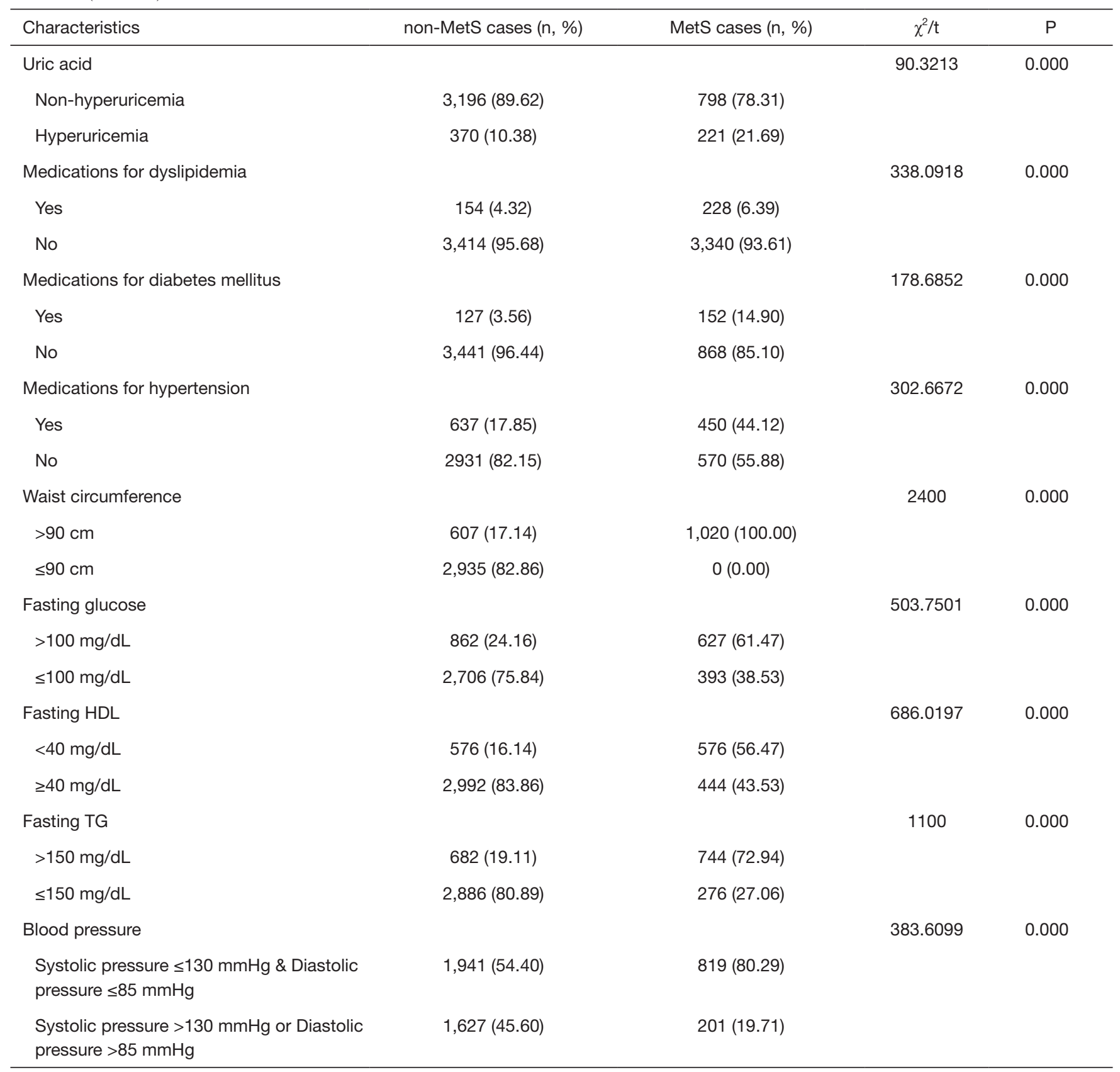

MetS, metabolic syndrome; TG, total triglyceride; HDL, high-density lipoprotein.

\section{Correlation between MetS and LUTS/BPH}

Four models were built to evaluate the association between MetS and LUTS/BPH (shown in Figure 3). In model 1 with no adjustment, patients with MetS had a 1.49 times higher risk of LUTS/BPH than patients without MetS $(95 \%$ CI, 1.23-1.81, $\mathrm{P}<0.001)$. After adjustments were made for demographic variables and individual behaviors, including age, smoking, alcohol consumption, geographical region, afternoon napping, sleeping time, depression, and marital status, the odds ratio $(\mathrm{OR})$ value decreased to 1.46 , but was still statistically significant $(\mathrm{P}<0.001)$. In model 3 , patients with MetS had a 1.47 times higher probability of LUTS/ 

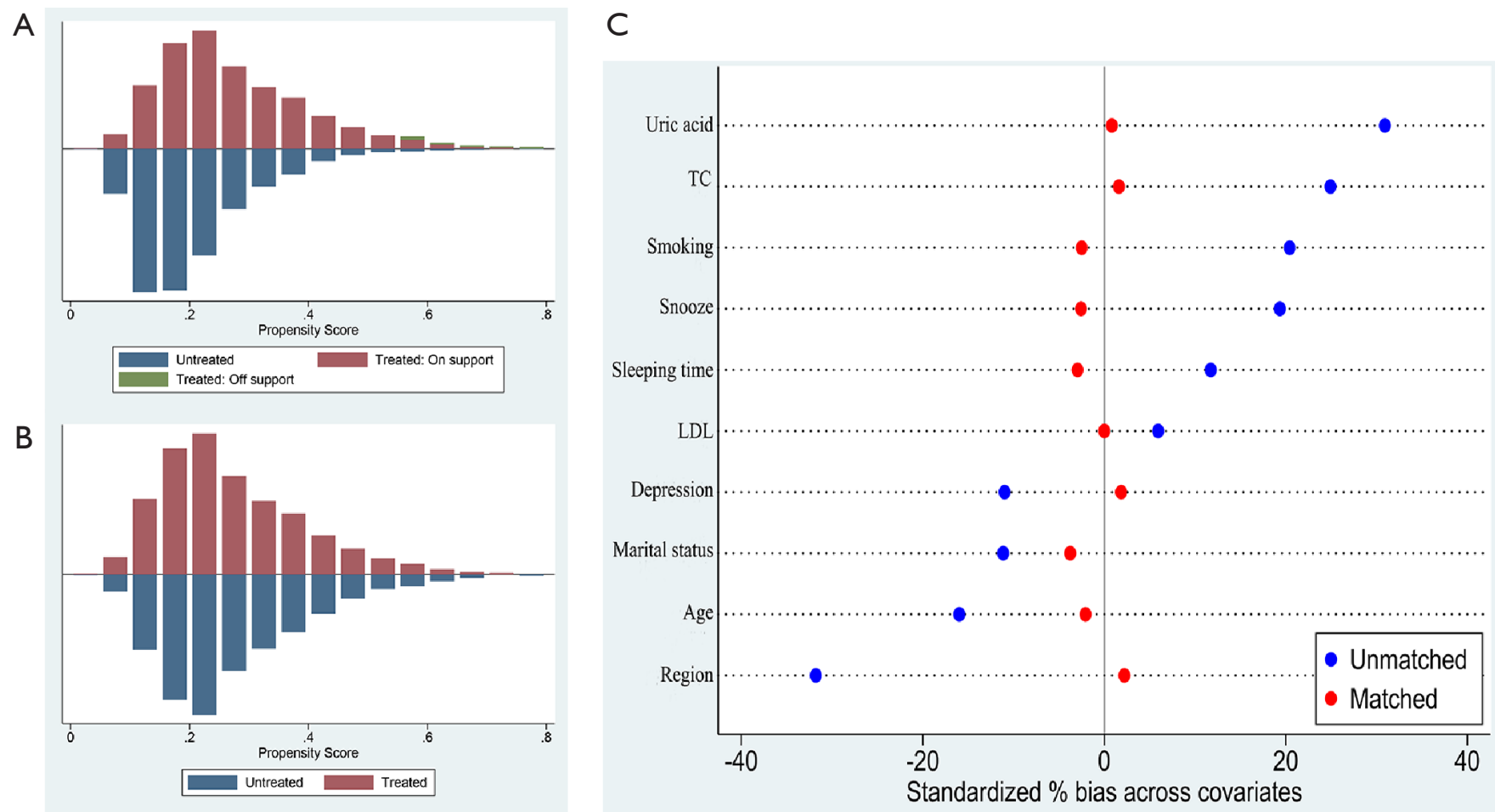

Figure 2 Propensity scores and absolute standardized differences. (A) shows the distribution of propensity before PSM. (B) shows the distribution of propensity after PSM. (C) shows the absolute standardized differences between the MetS and non-MetS groups. TC, total cholesterol; LDL, low-density lipoprotein; PSM, propensity score matching; MetS, metabolic syndrome.

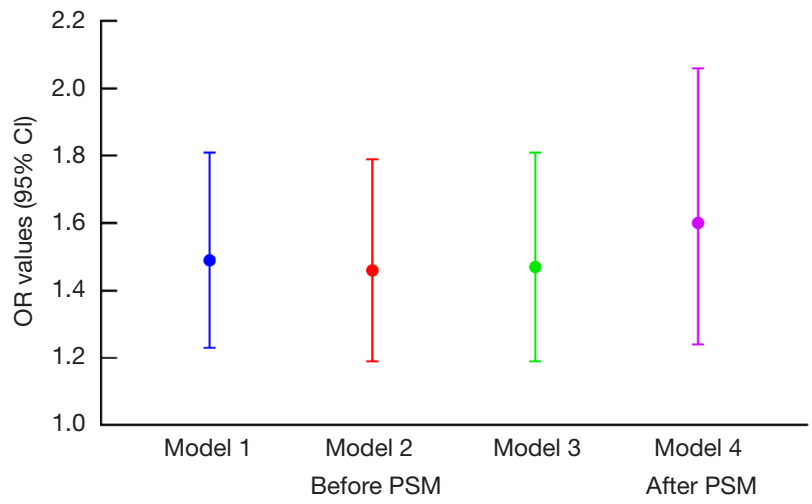

Figure 3 Logistic regression between MetS and LUTS/BPH before and after PSM. Model 1: adjusted for no variables. Model 2: Adjusted for age, smoking, alcohol consumption, geographic region, afternoon napping, sleeping time, depression, and marital status. Model 3: adjusted for Model 2 variables in addition to uric acid, LDL, and TC. Model 4: adjusted for no other variables. OR, odds ratios; PSM, propensity score matching; MetS, metabolic syndrome; LUTS/BPH, lower urinary tract symptoms suggestive of benign prostate hyperplasia; LDL, low-density lipoprotein; TC, total cholesterol.
BPH than non-MetS patients (95\% CI, 1.19-1.81, $\mathrm{P}<0.001$ ) after adjustments were made for all demographic variables and individual behaviors, in addition to uric acid, LDL, and TC. After PSM, the logistic regression results indicated that MetS subjects had a 1.60 times higher likelihood of LUTS/ $\mathrm{BPH}$ than non-MetS participants (95\% CI, 1.24-2.06, $\mathrm{P}<0.001)$.

\section{Correlation between LUTS/BPH and the constituents of MetS}

Logistic regression analysis produced inconsistent findings before and after PSM (see Figure 4). Although the OR values of HDL, visceral adiposity, and triglycerides were different before and after PSM, they were all statistically significant $(\mathrm{P}<0.05)$. However, glucose exhibited the opposite trend. Before PSM, no risk was observed (OR $=1.05, \mathrm{P}=0.622$ ), while after PSM, the OR value surged to 1.60 (95\% CI, 1.24-2.06, $\mathrm{P}<0.000$ ). Moreover, hypertension exhibited no risk for LUTS/BPH both before and after PSM $(\mathrm{P}>0.05)$. 


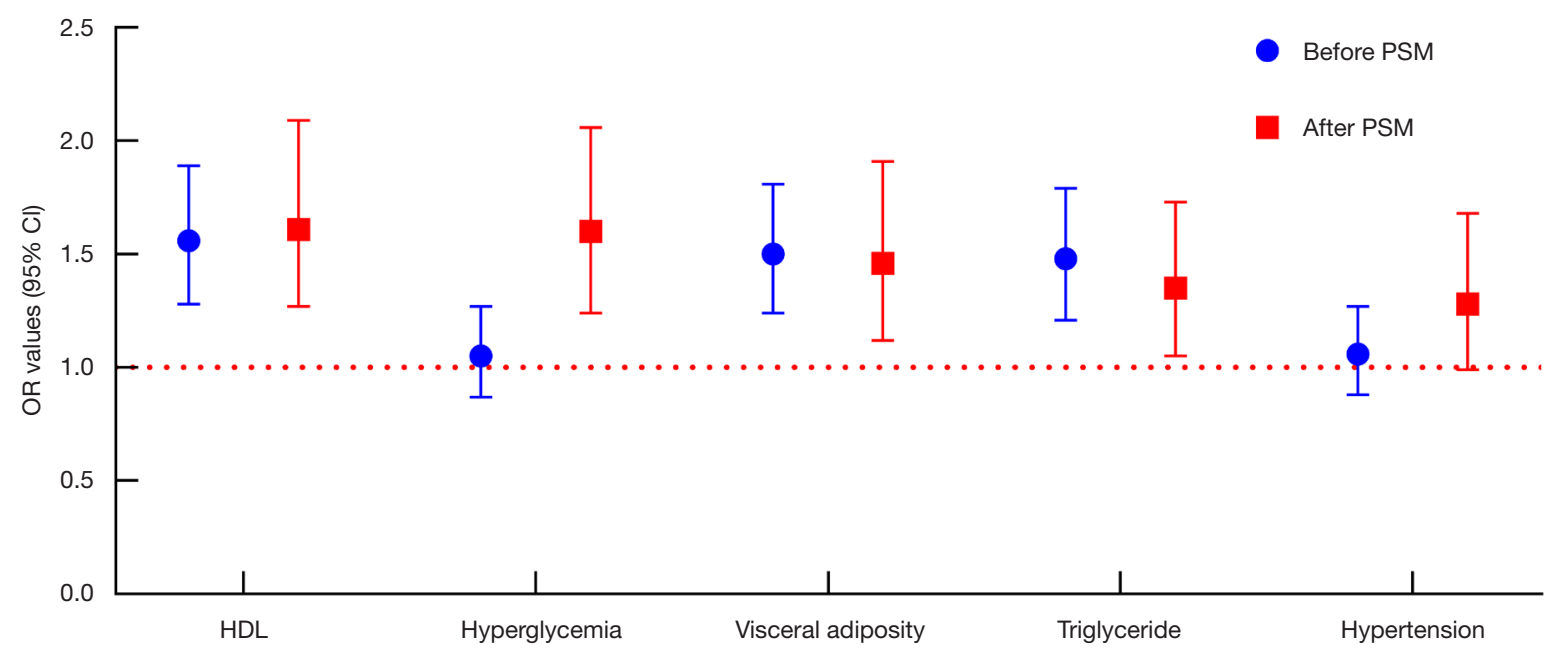

Figure 4 Logistic regression between LUTS/BPH and constituents of MetS. Before PSM, all of the logistic regressions were adjusted for age, smoking, alcohol consumption, geographic region, afternoon napping, sleeping time, depression, marital status, uric acid, LDL, and TC. After PSM, univariate logistic regression was employed. OR, odds ratios; PSM, propensity score matching; HDL, high-density lipoprotein; LUTS/BPH, lower urinary tract symptoms suggestive of benign prostate hyperplasia; MetS, metabolic syndrome; LDL, lowdensity lipoprotein; TC, total cholesterol; .

\section{Subgroup analyses}

Further logistic regression was employed to evaluate the association between these subgroups (see Figure 5). It was revealed that only MetS participants in the 50-60 year age group had a higher risk of LUTS/BPH $(\mathrm{OR}=2.15$, $\mathrm{P}<0.01)$. For MetS patients in the 40-50, 60-70, and $>70$ year age groups, this increased risk was not observed $(\mathrm{P}>0.05)$. Furthermore, this increased risk was also detected in participants who were married/cohabiting, slept fewer than 8 hours, settled in rural regions, had quit smoking, consumed alcohol more than once a month, had normal $\mathrm{TC}$, and did not have hyperuricemia $(\mathrm{P}<0.05)$. Interestingly, stratification based on depression, afternoon napping, and LDL was inconsequential; all participants displayed higher odds of LUTS/BPH, although with different ORs.

\section{Discussion}

The aging population trend presents significant challenges to healthcare, particularly in China, with its nearly 111 million citizens aged 65 years and above (28). In the aging population, both MetS and LUTS/BPH typically occur together, and there is currently a pressing need for further exploration to elucidate the association between them. CHARLS, a project specifically aimed at the elderly population, provides an opportunity to do so.
In the present study, all of the regression models indicated that MetS patients have a higher probability of LUTS/BPH. Previous studies aimed at the Chinese male population have reported similar findings $(11,13)$. Moreover, comparable conclusions have also been proposed in some U.S. and Asian populations. However, some similar studies in Asian populations report conflicting conclusions. In South Korean (19) and Japanese (21) studies, as well as in a study by Yang et al. (29), preliminary associations have been observed. Inconsistent conclusions have been reported across virtually all countries and ethnicities, particularly because of differences in lifestyle, economic status, sample size, study design, and sample population, thus making it difficult to explain the related phenomena using one or two factors. The inconsistent definition of MetS is one of the pivotal factors that should be given particular attention. Three main definitions are commonly employed, namely, those offered by the NCEP-ATP III, IDF, and WHO, although other local definitions of MetS, such as the definition used in the Hallym Aging Study (30) in Korea, are also commonly employed. This lack of uniformity can lead to different diagnoses for MetS patients. In our study, the IDF definition was adopted since it takes into consideration ethnic differences, has lower cutoff values, and is more suitable for the Chinese population, which may explain why an underlying positive association was 


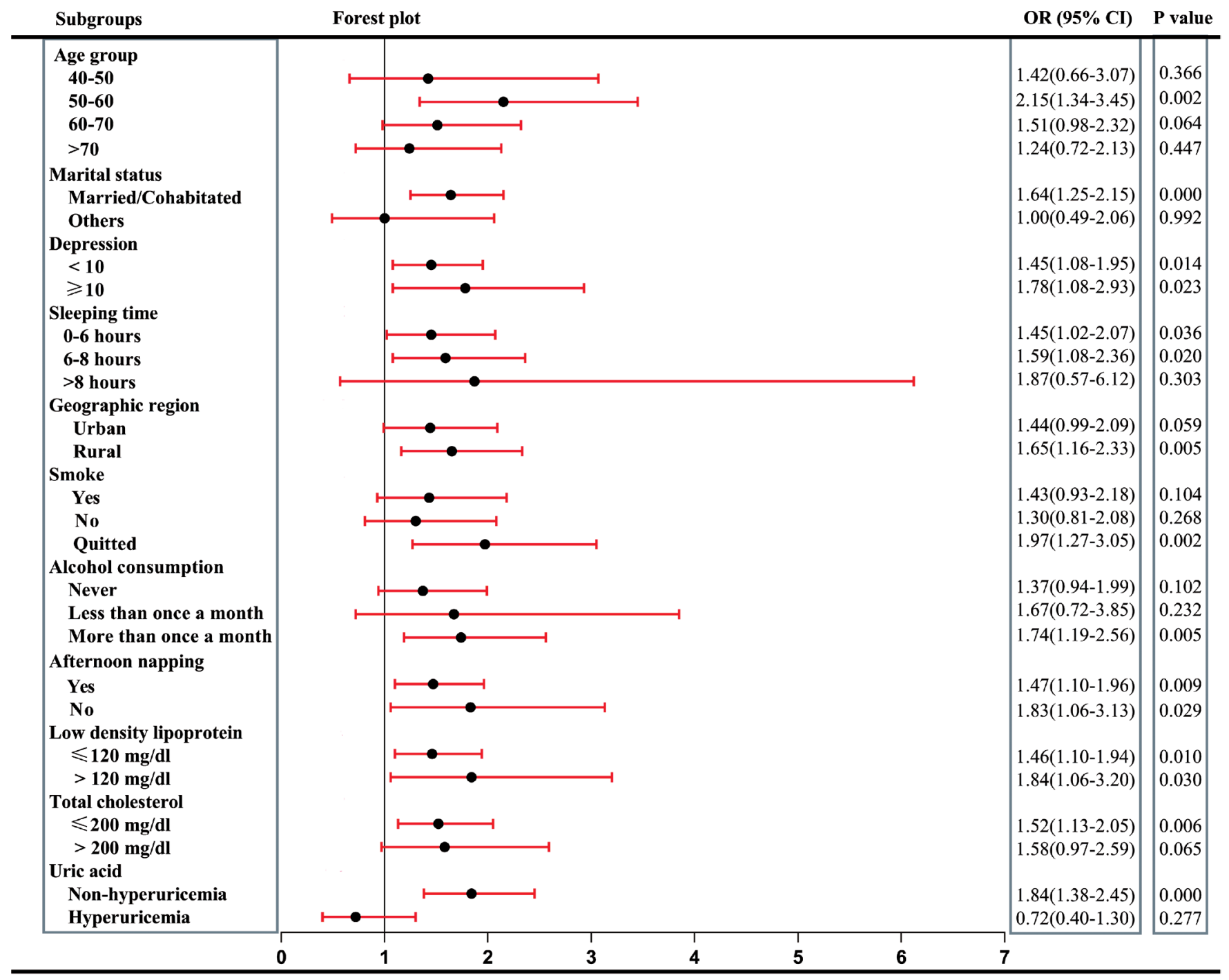

Figure 5 Results of subgroups analyses. OR, odds ratio; CI, confidence interval.

observed.

PSM was adopted in the present study to balance the covariates and reduce their interference in the estimation of outcome effects. PSM demonstrated that low HDL, hyperglycemia, visceral adiposity, and increased triglyceride levels are positively correlated with LUTS/BPH, which is in accordance with the findings of previous studies $(14,31)$. Visceral adiposity is a widely reported comorbidity with LUTS/BPH $(32,33)$, which was also observed in this study. Mondul et al. reported that participants with waistlines $>42$ inches have higher hazard ratios of 1.39 compared to subjects whose waistlines are $<33$ inches (32). Gacci et al. also noted that central obesity is associated with a higher risk of persistent storage LUTS after simple open prostatectomy or transurethral resection of the prostate (33). These studies indicate that obesity might be a therapeutic target for LUTS/BPH patients. Notably, obesity is also commonly observed with hyperglycemia via insulin resistance, a term to describe the inability of insulin to maintain glucose homeostasis (34). These can jointly contribute to LUTS/BPH, which may explain why hyperglycemia and central obesity exhibit close ties with LUTS/BPH here. Previous studies suggest that this close relationship may be directed by the systemic inflammation and oxidative stress commonly seen in obese individuals (35), which aid the growth of the prostate and lead to LUTS/ 


\section{BPH (36).}

Our study also found that the participants with low HDL or increased triglyceride levels had a greater likelihood of LUTS/BPH, which further supports an emerging understanding of the dyslipidemia profile in aging Chinese males. Similar results have also been reported in other studies $(37,38)$. Martin et al.'s results suggest that HDL cholesterol and lower triglycerides contribute to predicting the improvement of LUTS (37). Chiu et al.'s study enrolled 20,152 patients with benign prostatic enlargement, and concluded that hyperlipidemia is significantly positively correlated with benign prostatic enlargement (38). In contrast, one previous study observed no relationship between serum lipid levels and LUTS/ $\mathrm{BPH}$ is observed (39). This conclusion may be biased due to its small sample size of 257 males aged 40 years and above, restricted to a single village in Turkey. Our subgroup analysis results also indicated that LDL, which is not a component of MetS, also increases the risk of LUTS/ BPH. A prospective, community-based cohort study revealed a similar conclusion: elevated LDL increases the risk of $\mathrm{BPH}$ in diabetic men fourfold, but not in the overall cohort (40). According to previous publications $(41,42)$, this phenomenon may be mediated by inflammatory stimulation between altered lipid metabolism and LUTS/BPH. In a MetS model obtained by feeding male rabbits a high-fat diet for 12 weeks, prostate inflammation was detected. The mRNA expression of several proinflammatory markers, such as interleukin (IL)-8, IL-6, IL-1 $\beta$, and tumor necrosis factor (TNF)- $\alpha$, was significantly increased in the prostates of these rabbits (41). Inflammation in the prostate leads to the cell proliferation and tissue remodeling in $\mathrm{BPH}$ (42). Hence, altered lipid metabolism may initially activate inflammation, and in turn further enlarge the prostate.

However, hypertension did not lead to an increased risk in our study, both before and after PSM. Rohrmann et al. reported that hypertension was found to be positively corrected with LUTS (43). Also, Roehrborn et al.'s study indicated that hypertension is the most common comorbidity in LUTS/BPH patients (44). In contrast, results from the Massachusetts Male Aging Study demonstrated conflicting findings (45). Rohrmann et al.'s study was conducted with 2,372 men aged 60 years and older, while the Massachusetts Male Aging Study was performed with 1,709 men aged 40-70 years. It is widely accepted that with aging, the prevalence of hypertension and LUTS/BPH increases (46). The discrepancies between the sampling populations in the different age groups might have led to the inconsistent conclusions. The present study was conducted with males aged 40 years and above, and the conclusion is consistent with that of the Massachusetts Male Aging Study, although further prospective cohort studies are needed to verify the findings.

Interestingly, the subgroup analyses revealed that statistical significance was only present in the participants aged 50-60 years old, which seems to be in line with the report from the Boston Area Community Health Survey, which reported that only the MetS patients aged $<60$ years old had a higher probability of LUTS/BPH (47). This may be attributable to the fact that males in different age ranges exhibit different risk factors and the influences of these risk factors for different age ranges vary. Our results were primarily analyzed by regression analyses, which relied on their covariates to adjust the final $\mathrm{OR}$ and $\mathrm{P}$ values. Hence, OR values after adjustments are different for them. Moreover, from a statistical perspective, the subgroup analyses suggested that males aged 50-60 years may be a high-risk population that requires greater healthcare, although this needs further validation by prospective cohort study.

Certain limitations to this study should also be noted. First, although some potential confounders were considered in the analyses, others, such as educational status and income, were not included, and might have introduced some bias into the study. Second, the present study adopted a more convincing method, PSM, to examine the association between MetS and LUTS/BPH. However, randomization by PSM cannot identify the causal effect between MetS and LUTS/BPH; only a high-quality prospective cohort study can explore causality, which is what we plan to do in the future.

As the aging trend continues, LUTS/BPH will markedly affect more males and place substantial strains on healthcare resources. Thus, prevention of LUTS/BPH remains critically important to public health. Our study indicates that MetS is significantly correlated with LUTS/BPH in aging males. In a society with a high incidence of metabolic diseases, acting on MetS in the early stages and promoting a healthy lifestyle in aging males will be instrumental in containing the occurrence of LUTS/BPH and reducing the adverse consequences.

\section{Conclusions}

Patients with MetS had a higher probability of LUTS/ $\mathrm{BPH}$ in the aging Chinese male population. Our results 
demonstrated that patients who had low HDL, abdominal obesity, high triglyceride levels, or hyperglycemia had an increased risk of LUTS/BPH; however, this was not the case for hypertensive patients.

\section{Acknowledgments}

The authors express thanks to the office of CHARLS.

Funding: This work was supported by the Natural Science Foundation of China (81871147 \& 81671453), Sichuan Science and Technology Program (2018TJPT0018), and Chengdu Science and Technology Program (2019-YFYF00087-SN).

\section{Footnotes}

Reporting Checklist: The authors have completed the STROBE reporting checklist. Available at http://dx.doi. org/10.21037/tau-20-1127

Conflicts of Interest: All authors have completed the ICMJE uniform disclosure form (available at http://dx.doi. org/10.21037/tau-20-1127). The authors have no conflicts of interest to declare.

Ethical Statement: The authors are accountable for all aspects of the work in ensuring that questions related to the accuracy or integrity of any part of the work are appropriately investigated and resolved. The study was conducted in accordance with the Declaration of Helsinki (as revised in 2013). This study was reviewed and approved by the ethics committee of Peking University (IRB 0000105211014). Written and oral informed consent was obtained from all participants prior to their enrollment in this study.

Open Access Statement: This is an Open Access article distributed in accordance with the Creative Commons Attribution-NonCommercial-NoDerivs 4.0 International License (CC BY-NC-ND 4.0), which permits the noncommercial replication and distribution of the article with the strict proviso that no changes or edits are made and the original work is properly cited (including links to both the formal publication through the relevant DOI and the license). See: https://creativecommons.org/licenses/by-nc-nd/4.0/.

\section{References}

1. Madersbacher S, Alivizatos G, Nordling J, et al. EAU 2004 guidelines on assessment, therapy and follow-up of men with lower urinary tract symptoms suggestive of benign prostatic obstruction (BPH guidelines). Eur Urol 2004;46:547-54.

2. Langan RC. Benign Prostatic Hyperplasia. Prim Care 2019;46:223-32.

3. Xiong Y, Zhang Y, Li X, et al. The prevalence and associated factors of lower urinary tract symptoms suggestive of benign prostatic hyperplasia in aging males. Aging Male 2020;1-8.

4. Yue L, Wang T, Ge Y, et al. Prevalence and heritability of benign prostatic hyperplasia and LUTS in men aged 40 years or older in Zhengzhou rural areas. Prostate 2019;79:312-9.

5. Lee AJ, Garraway WM, Simpson RJ, et al. The natural history of untreated lower urinary tract symptoms in middle-aged and elderly men over a period of five years. Eur Urol. 1998;34:325-32.

6. Shih HJ, Huang CJ, Lin JA, et al. Hyperlipidemia is associated with an increased risk of clinical benign prostatic hyperplasia. Prostate 2018;78:113-20.

7. La Vignera S, Condorelli RA, Russo GI, et al. Endocrine control of benign prostatic hyperplasia. Andrology 2016;4:404-11.

8. Vignozzi L, Gacci M, Maggi M. Lower urinary tract symptoms, benign prostatic hyperplasia and metabolic syndrome. Nat Rev Urol 2016;13:108-19.

9. Sebastianelli A, Gacci M. Current Status of the Relationship Between Metabolic Syndrome and Lower Urinary Tract Symptoms. Eur Urol Focus 2018;4:25-7.

10. Kasturi S, Russell S, McVary KT. Metabolic syndrome and lower urinary tract symptoms secondary to benign prostatic hyperplasia. Curr Urol Rep 2006;7:288-92 .

11. Pan JG, Liu M, Zhou X. Relationship between lower urinary tract symptoms and metabolic syndrome in a Chinese male population. J Endocrinol Invest 2014;37:339-44.

12. Lu J, Wang L, Li M, et al. Metabolic Syndrome Among Adults in China: The 2010 China Noncommunicable Disease Surveillance. J Clin Endocrinol Metab 2017;102:507-15.

13. Fu Y, Zhou Z, Yang B, et al. The Relationship between the Clinical Progression of Benign Prostatic Hyperplasia and Metabolic Syndrome: A Prospective Study. Urol Int 2016;97:330-5.

14. Gacci M, Vignozzi L, Sebastianelli A, et al. Metabolic syndrome and lower urinary tract symptoms: the role of inflammation. Prostate Cancer Prostatic Dis 
2013;16:101-6.

15. Park YW, Kim SB, Kwon H, et al. The relationship between lower urinary tract symptoms/benign prostatic hyperplasia and the number of components of metabolic syndrome. Urology 2013;82:674-9.

16. Grzesiak K, Rył A, Baranowska-Bosiacka I, et al. Comparison between selected hormone and protein levels in serum and prostate tissue homogenates in men with benign prostatic hyperplasia and metabolic disorders. Clin Interv Aging 2018;13:1375-82.

17. Rył A, Rotter I, Miazgowski T, et al. Metabolic syndrome and benign prostatic hyperplasia: association or coincidence? Diabetol Metab Syndr 2015;7:94.

18. Ozden C, Ozdal OL, Urgancioglu G, et al. The correlation between metabolic syndrome and prostatic growth in patients with benign prostatic hyperplasia. Eur Urol 2007;51:199-206.

19. Park YW, Min Sk, Lee JH. Relationship between Lower Urinary Tract Symptoms/Benign Prostatic Hyperplasia and Metabolic Syndrome in Korean Men. World J Mens Health 2012;30:183-8.

20. Gao Y, Wang M, Zhang H, et al. Are metabolic syndrome and its components associated with lower urinary tract symptoms? Results from a Chinese male population survey. Urology 2012;79:194-201.

21. Ohgaki K, Hikima N, Horiuchi K, et al. Association between metabolic syndrome and male lower urinary tract symptoms in Japanese subjects using three sets of criteria for metabolic syndrome and International Prostate Symptom Score. Urology 2011;77:1432-8.

22. Gacci M, Corona G, Vignozzi L, et al. Metabolic syndrome and benign prostatic enlargement: a systematic review and meta-analysis. BJU Int 2015;115:24-31.

23. Zhao Y, Hu Y, Smith JP, et al. Cohort profile: the China Health and Retirement Longitudinal Study (CHARLS). Int J Epidemiol 2014;43:61-8.

24. Andresen EM, Byers K, Friary J, et al. Performance of the 10-item Center for Epidemiologic Studies Depression scale for caregiving research. SAGE Open Med 2013;1:2050312113514576.

25. Taguchi I, Iimuro S, Iwata H, et al. High-Dose Versus Low-Dose Pitavastatin in Japanese Patients With Stable Coronary Artery Disease (REAL-CAD): A Randomized Superiority Trial. Circulation 2018;137:1997-2009.

26. Kanjanavanit S, Puthanakit T, Vibol U, et al. High prevalence of lipid abnormalities among antiretroviralnaive $\mathrm{HIV}$-infected Asian children with mild-to-moderate immunosuppression. Antivir Ther 2011;16:1351-5.
27. Li Q, Li X, Wang J, et al. Diagnosis and treatment for hyperuricemia and gout: a systematic review of clinical practice guidelines and consensus statements. BMJ Open 2019;9:e026677.

28. Zeng Y. Towards Deeper Research and Better Policy for Healthy Aging --Using the Unique Data of Chinese Longitudinal Healthy Longevity Survey. China Economic J 2012;5:131-49.

29. Yang TK, Hsieh JT, Chen SC, et al. Metabolic syndrome associated with reduced lower urinary tract symptoms in middle-aged men receiving health checkup. Urology 2012;80:1093-7.

30. Lee SH, Lee SK, Choo MS, et al. Relationship between Metabolic Syndrome and Lower Urinary Tract Symptoms: Hallym Aging Study. Biomed Res Int 2015;2015:130917.

31. Parsons JK. Modifiable risk factors for benign prostatic hyperplasia and lower urinary tract symptoms: new approaches to old problems. J Urol 2007;178:395-401.

32. Mondul AM, Giovannucci E, Platz EA. A prospective study of obesity, and the incidence and progression of lower urinary tract symptoms. J Urol 2014;191:715-21.

33. Gacci M, Sebastianelli A, Salvi M, et al. Central obesity is predictive of persistent storage lower urinary tract symptoms (LUTS) after surgery for benign prostatic enlargement: results of a multicentre prospective study. BJU Int 2015;116:271-7.

34. Könner AC, Brüning JC. Selective insulin and leptin resistance in metabolic disorders. Cell Metab 2012;16:144-52.

35. Furukawa S, Fujita T, Shimabukuro M, et al. Increased oxidative stress in obesity and its impact on metabolic syndrome. J Clin Invest 2004;114:1752-61.

36. Chughtai B, Forde JC, Thomas DD, et al. Benign prostatic hyperplasia. Nat Rev Dis Primers 2016;2:16031.

37. Martin S, Lange K, Haren MT, et al. Risk factors for progression or improvement of lower urinary tract symptoms in a prospective cohort of men. J Urol 2014;191:130-7.

38. Chiu YL, Kao S, Lin HC, et al. Benign prostatic enlargement is not associated with diabetes: a populationbased study. Andrology 2015;3:933-6.

39. Lekili M, Müezzinoğlu T, Uyanik BS, et al. Serum lipid levels in benign prostatic hyperplasia. World J Urol 2006;24:210-3.

40. Parsons JK, Bergstrom J, Barrett-Connor E. Lipids, lipoproteins and the risk of benign prostatic hyperplasia in community-dwelling men. BJU Int 2008;101:313-8.

41. Vignozzi L, Morelli A, Sarchielli E et al. Testosterone 
protects from metabolic syndrome-associated prostate inflammation: an experimental study in rabbit. J Endocrinol 2012;212:71-84.

42. Vignozzi L, Rastrelli G, Corona G et al. Benign prostatic hyperplasia: a new metabolic 1. disease? J Endocrinol Invest 2014;37:313-22.

43. Rohrmann S, Smit E, Giovannucci E, et al. Association between markers of the metabolic syndrome and lower urinary tract symptoms in the Third National Health and Nutrition Examination Survey (NHANES III). Int J Obes (Lond) 2005;29:310-6.

44. Roehrborn CG, Nuckolls JG, Wei JT, et al. The benign prostatic hyperplasia registry and patient survey: study

Cite this article as: Xiong Y, Zhang Y, Tan J, Qin F, Yuan J. The association between metabolic syndrome and lower urinary tract symptoms suggestive of benign prostatic hyperplasia in aging males: evidence based on propensity score matching. Transl Androl Urol 2021;10(1):384-396. doi: 10.21037/tau-201127 design, methods and patient baseline characteristics. BJU Int 2007;100:813-9.

45. Meigs JB, Mohr B, Barry MJ, et al. Risk factors for clinical benign prostatic hyperplasia in a communitybased population of healthy aging men. J Clin Epidemiol 2001;54:935-44.

46. Buford TW. Hypertension and aging. Ageing Res Rev 2016;26:96-111.

47. Kupelian V, McVary KT, Kaplan SA, et al. Association of lower urinary tract symptoms and the metabolic syndrome: results from the Boston Area Community Health Survey. J Urol 2013;189:S107-14; discussion S115-6. 